\title{
Implementaciones en Hardware de técnicas de Radiogoniometría
}

\section{Hardware Implementations based on Radio Direction Finding}

\author{
Tibisay Sánchez ${ }^{1}$, Alfredo David Redondo ${ }^{2}$, Andrés Felipe García ${ }^{3}$, Cristina Gómez ${ }^{4}$, \\ Leonardo Betancur ${ }^{5}$ y Roberto Carlos Hincapié ${ }^{6}$
}

\begin{abstract}
Resumen
En este artículo se presenta una revisión bibliográfica y un análisis comparativo de implementaciones en hardware de técnicas de Radiogoniometría, también conocidas como Radio Direction Finding (RDF), que permiten identificar la mejor opción para implementar estas funcionalidad en actividades de gestión del espectro en países en vía de desarrollo. Dentro de las implementaciones tratadas se incluyen técnicas clásicas como Pseudo-Doppler y técnicas avanzadas de alta resolución como MUSIC. Se presentan diferentes alternativas de hardware para realizar las implementaciones las cuales incluyen SDR (Software Defined Radio), FPGA (Field Programmable Gate Array) y DSP (Digital Signal Processor); a la vez que se incluyen algunas configuraciones híbridas dónde se mezcla el software y el hardware con el fin de optimizar recursos de tiempo y dinero. Adicionalmente se muestran algunas aplicaciones comerciales que emplean técnicas de geolocalización basadas en información de ángulos de llegada, tiempos de llegada u otros parámetros que permiten realizar el proceso de triangulación o trilateración según sea el caso.
\end{abstract}

Palabras clave: Radiogoniometría; radiolocalización; ángulo de llegada (AoA); diferencia de tiempos de llegada (TDoA); implementación, radio definido por software (SDR); arreglos de compuertas programables por campo (FPGA); procesador digital de señales (DSP).

\begin{abstract}
This paper presents a survey and a comparison between different radio direction finding implementations, leading to identify the best option for implementation of this feature in spectrum management activities for developing countries. The implementations analyzed include classic techniques like Pseudo-Doppler and advanced high-resolution techniques like MUSIC. Different hardware alternatives to implement the algorithms are presented, including SDR (Software Defined Radio), FPGA (Field Programmable Gate Array) and DSP (Digital Signal Processing). Also some hybrid configurations are included, where software and hardware are

1 Universidad Pontificia Bolivariana, Medellín, tibisay.sanchez@upb.edu.co, $\mathrm{MsC}(\mathrm{c})$

2 Universidad Pontificia Bolivariana, Medellín, alfredodavid.redondo@upb.edu.co, MsC(c)

3 Universidad Pontificia Bolivariana, Medellín, andresf.garciare@upb.edu.co, $\mathrm{MsC}(\mathrm{c}), \mathrm{Ph} . \mathrm{D}(\mathrm{c})$

4 Universidad Pontificia Bolivariana, Medellín, cristina.gomez@upb.edu.co, Ph.D

5 Universidad Pontificia Bolivariana, Medellín, leonardo.betancur@upb.edu.co, Ph.D

6 Universidad Pontificia Bolivariana, Medellín, roberto.hincapie@upb.edu.co, Ph.D
\end{abstract}


combined aiming to optimize time and cost resources. Finally some commercial applications are shown. These use angle of arrival, time difference of arrival and other parameters for triangulation or trilateration purposes.

Key words: Direction finding (DF); radiolocalization; angle of arrival (AoA), time difference of arrival (TDoA); implementation; software defined radio (SDR); field programmable gate array (FPGA); digital signal processor (DSP).

\section{Introducción}

La creciente demanda de servicios de comunicaciones inalámbricas y el constante desarrollo que ha tenido este sector de las telecomunicaciones obliga a las entidades reguladoras a mejorar la gestión y la vigilancia del recurso fundamental para este tipo de comunicaciones: el espectro radioeléctrico.

Hoy en día una de las exigencias que hace la Unión Internacional de Telecomunicaciones (UIT) en el Manual de Comprobación técnica del Espectro (UIT, 2011) y en las recomendaciones ITU-T k.83 (ITU, 2011) y k.91 (aún sin publicar formalmente) consiste en realizar mediciones de las radiaciones no-ionizantes (RNI), además de la localización de las fuentes emisoras de las mismas, bien sean fuentes legales e ilegales. Para completar esta última tarea se emplean técnicas de Radiogoniometría, más conocidas como Radio Direction Finding (RDF) (Van Trees, 2004), algunas de ellas clásicas como Watson-Watt, Pseudo Doppler y Correlative Interferometer explicadas en (Keaveny, 2005), y otras más avanzadas como MUSIC, ESPRIT, entre otras (Gross, 2015). Dichas técnicas requieren de un sistema que permita realizar todo el procesamiento de la información de tal manera que se pueda obtener una estimación final de la posición del emisor. Todo sistema de geolocalización inalámbrica cuenta con tres elementos básicos: un dispositivo de sensado, un algoritmo de posicionamiento y un módulo que permita visualizar los resultados obtenidos (Roxin, et al., 2007).

El dispositivo de sensado puede ser cualquier plataforma de hardware que permita la recepción de señales de radiofrecuencia y su procesamiento, como es el caso de las FPGA (XILINX, s.f.), los DSP (Bahamondes, 2009) o los SDR (Fette, 2006). El módulo de visualización puede ser tan complejo o tan simple como se requiera, por ejemplo, un computador (PC) puede desempeñar esta función sin tener problema alguno. Finalmente el algoritmo de posicionamiento es lo que marca la diferencia entre un sistema y otro ya que depende del entorno de radio que se está evaluando.
Todas las técnicas tienen ventajas y limitaciones que son determinantes en el momento de la estimación final.

En general las técnicas de posicionamiento operan bajo dos principios básicos: triangulación o trilateración (Roxin, et al., 2007). En cada uno de estos principios existen alternativas que emplean diferente tipo de información de la señal para estimar la posición del emisor, por ejemplo, información de ángulo de llegada (AoA) (Gross, 2015), tiempos de llegada (ToA) o diferencia de tiempos de llegada (TDoA) (Zekavat \& Buehrer, 2011), entre otras.

Sin embargo como se mencionó previamente, existen limitantes en las técnicas de posicionamiento. En el caso de AoA su principal inconveniente es la alta sensibilidad a los efectos de la multitrayectoria, además de las exigencias en cuanto al arreglo de antenas (EMT, 2004), (Series, 2011). Por su parte ToA y TDoA son técnicas que exigen al menos tres sensores que permitan triangular la posición del emisor, y además estos deben estar completamente sincronizados en tiempo para obtener buenos resultados (Series, 2011).

El siguiente documento tiene como fin presentar un estado del arte sobre las implementaciones de técnicas de RDF, explorando desarrollos sobre FPGAs, DSP, SDR u otras opciones como plataformas de hardware.

El estudio realizado se propone mostrar prototipos experimentales y su desempeño, especialmente en ambientes de laboratorio, ya que apuntan a desarrollos viables que sean de bajo costo y que permitan cumplir con las exigencias mínimas hechas por ITU en el Manual de Comprobación Técnica del Espectro para países en vías de desarrollo. Las directrices marcadas por ITU para este tipo de territorios acceden a tener menor resolución en cuanto a la precisión en la estimación realizada, lo cual permite emplear plataformas más económicas y por lo general abiertas, dando relevancia a estos trabajos de investigación con miras a crear productos comerciales que se puedan emplear en países como Colombia. Un caso puntual de este tipo de iniciativas es el SMS4DC (ITU, s.f.), el cual 
cumple con funciones de sensado de espectro y permite integración rápida con otras plataformas, especialmente con SDR, brindando posibilidades de rápida configuración (Navarro, s.f.). Es importante tener en cuenta que a pesar de tener técnicas de alta resolución y técnicas clásicas, todas ellas pueden ser implementadas en cualquier plataforma de hardware, sin embargo, la elección de dicha plataforma influirá en el desempeño total del sistema. Lo anterior permite concluir que las diferencias entre países en vías de desarrollo o países desarrollados radican en el hardware empleado y no en la técnica utilizada.

El documento está estructurado de la siguiente manera: una primera sección que contiene las implementaciones sobre SDR de técnicas de RDF. Dentro de esta sección se encuentran las primeras implementaciones de la historia de estos algoritmos y algunas aplicaciones industriales. Adicionalmente se muestran prototipos académicos clasificados y agrupados por el algoritmo implementado. La sección II incluye implementaciones en hardware sobre otras plataformas alternativas: dentro de las que se incluyen FPGA, DSP, configuraciones híbridas que incluyen desarrollos en hardware y software, y otras plataformas. De igual manera se presentan las implementaciones agrupadas por algoritmos. Las conclusiones del artículo se presentan en otra sección, y finalmente se presentan las referencias bibliográficas consultadas para la redacción del presente artículo.

\section{Implementaciones de técnicas de RDF sobre SDR}

Actualmente se cuenta con una regulación estricta en cuanto al monitoreo del espectro radioeléctrico, tomando en cuenta no solo los niveles de potencia o interferencia, sino también localización de emisiones legales e ilegales. Para ello se han desarrollado implementaciones en hardware de algoritmos de RDF, buscando diferentes alternativas como arreglos de antena o técnicas Multiple Input Multiple Output (MIMO). Sin embargo, uno de los mayores inconvenientes que tienen este tipo de sistemas es su alto nivel de complejidad computacional, especialmente cuando se emplean técnicas MIMO, motivo por el cual se han buscado alternativas de implementaciones más económicas como las plataformas definidas por software o SDR (Navarro, s.f.) por sus siglas en inglés.

\subsection{Inicios de RDF y aplicaciones industriales}

Los primeros usos de RDF datan del año 1900 cuando Fred Adcock presentó un arreglo de antenas de cuatro elementos que se empleaba para estimar la posición de una fuente transmisora. Posteriormente durante La II Guerra Mundial el uso de estas técnicas se incrementó debido a su potencial para estimar la posición de fuentes de comunicación enemigas y de esta manera generar ventaja sobre el enemigo (Stieber, 2012).

Uno de los sistemas más prometedores de la época fue el Wide-Aperture Direction Finding (WADF), el cual empleaba un arreglo circular de antenas conocido como CDAA (Circulary Disposed Antenna Array). Los CDAA eran arreglos de antenas de gran tamaño que empleaban monopolos dispuestos en una circunferencia con diámetros que oscilaban entre los $50 \mathrm{~m}$ y los $150 \mathrm{~m}$. En este tipo de arreglos, la selección del elemento de antena que recibe la señal se hace empleando un conmutador RF (Radio Frecuencia) que conmuta a gran velocidad los elementos del arreglo, captando en cada uno un valor diferente del ángulo azimutal, el cual se almacena y emplea posteriormente para la estimación de la posición del transmisor (Stieber, 2012).

En 1970, la guardia costera de Estados Unidos desarrolló el National Distress System (NDS), el cual contaba con un receptor de radio que operaba por encima de los $300 \mathrm{MHz}$ y estaba situado a lo largo de la costa americana cubriendo hasta 20 millas náuticas desde cualquier orilla. Los receptores estaban conectados a través de 44 centros de comunicación monitoreados constantemente. Posteriormente, empleando lo anterior como base surge el proyecto RESCUE 21 dentro del que se emplea un sistema de antenas VHF (Very High Frequency) con capacidades básicas de RDF. Sin embargo, sus principales problemas fueron el alto costo de instalación, la alta complejidad del sistema y su integración con otros sistemas (Stieber, 2012).

En (Stieber, 2012) se encuentran también algunas aplicaciones actuales que emplean RDF, como por ejemplo Civil Air Patrol (CAP), el cual es un equipo que presta apoyo a más del $85 \%$ de las misiones de búsqueda y rescate ejecutadas para la Fuerza Aérea de USA. CAP tiene un rango de operación entre $121.5 \mathrm{MHz}$ y $406 \mathrm{MHz}$ para ubicar aviones desaparecidos.

Adicionalmente en (Stieber, 2012) se presentan implementaciones de RDF para rescate de personas desaparecidas en catástrofes naturales, empleando Personal Locator Beacons (PLB) operando en 1.6 $\mathrm{GHz}$ y Avalanche Beacons que operan en $457 \mathrm{KHz}$. 
Además de las aplicaciones previamente mencionadas, en (Stieber, 2012) también se muestran implementaciones para rastreo de animales como Wildlife Tracking, cuyos dispositivos emplean frecuencias entre 30 - $400 \mathrm{MHz}$. Finalmente (Stieber, 2012) muestra el sistema de emergencias Wireless E911 perteneciente a la FCC (Federal Communications Comission), y obliga desde 2010 a todos los operadores celulares de USA a proporcionar información de localización en casos de emergencia, con una precisión entre 50 - 300 metros, para lo cual los operadores emplean métodos como A-GPS (Assited Global Positioning Sysem), TDOA, AoA y E-OTD (Enhanced Observed Time Difference).

\subsection{Prototipos académicos}

Lo anterior hace referencia a aplicaciones de RDF empleadas en la industria. A continuación se referenciarán entonces trabajos desarrollados en investigaciones académicas que buscan evaluar el desempeño de diferentes técnicas sobre plataformas consideradas de bajo costo.

\section{A. MUSIC}

La revisión del tema permite observar que la mayoría de las implementaciones de técnicas de RDF aplican algoritmos de DoA(Direction of Arrival) o AoA. Es el caso de (Hua, et al., 2011) que presenta una implementación que contiene 4 receptores de RF independientes que conforman un arreglo lineal uniforme (ULA) de 4 elementos de antena, un conversor análogo/digital y un módulo procesador de señal donde se implementa un algoritmo de calibración de fase. Se emplea MUSIC como algoritmo de AoA para la estimación y se prueba el funcionamiento de la plataforma dentro de una cámara anecoica, cuyos resultados son comparados posteriormente con simulaciones hechas en Mathworks Simulink.

Como trabajo complementario a (Hua, et al., 2011), en (Hua, et al., 2012) se emplea un arreglo de 16 dipolos que se organizan en 4 grupos de 4 elementos cada uno, formando 4 ULAs. Cada uno de los ULA tiene un rango de estimación de $90^{\circ}$, cubriendo los $360^{\circ}$ mediante la combinación de ellos. Los resultados de las pruebas permiten concluir que se obtienen buenos resultados en ambientes outdoor, incluso en presencia de multitrayectoria.

Otro de los trabajos que emplea MUSIC como algoritmo de estimación se presenta en (Tayem, et al., 2014), donde también se implementa ESPRIT. Ambos algoritmos se desarrollan sobre una plataforma PXI de National Instruments y se realiza toda la programación del hardware a través de LabView. El estimador de DoA emplea un chasis que incluye un controlador y módulos RF de transmisión y recepción, un módulo down-converter, digitalizadores y amplificadores RF. Se utiliza un ULA de cuatro elementos de antena omnidireccionales, con una separación de $16 \mathrm{~cm}$ entre ellos, y una distancia de $4.5 \mathrm{~m}$ entre transmisor y receptor. Las medidas se toman en un ambiente outdoor con el fin de emular un ambiente real. Teniendo en cuenta que existe una fuente ubicada con un ángulo de $100^{\circ}$ con respecto al ULA, el prototipo es capaz de ubicarla en $101.48^{\circ}$ para el caso de MUSIC y en $99.96^{\circ}$ cuando se emplea ESPRIT, concluyendo que el sistema tiene un buen desempeño.

\section{B. Root-MUSIC}

Por su parte, en (Zekavat, et al., 2007) se combinan dos algoritmos de AoA que permiten mitigar las debilidades de cada uno de ellos por separado y potencializar sus fortalezas: Delay and Sum (DAS) y Root-MUSIC. Este último se modifica con el fin de reducir su complejidad computacional obligándolo a que emplee información proporcionada por DAS, de tal manera que solo se emplee una raíz que se iguala a 1, con el valor de fase obtenido previamente por DAS. Los resultados obtenidos de la implementación en hardware son comparados con simulaciones de modelos Monte Carlo que emplean 4 elementos de antena, y se comparan parámetros de $\mathrm{SNR}$, número de antenas, potencia de la señal recibida e influencia de multitrayectoria sobre la estimación. Finalmente se concluye que la combinación de ambas alternativas permite reducir en gran medida el costo computacional del sistema.

\section{Pseudo-Doppler}

Adicional a las implementaciones comerciales mencionadas previamente, en (Stieber, 2012) también se desarrollan dos prototipos experimentales. El primero de ellos basado en la técnica clásica de PseudoDoppler, donde se emplea un DSP que permite comparar las frecuencias Doppler captadas por el sistema, a la vez que realiza la calibración y sincronización de las señales entrantes al conmutador RF, con las señales de referencia del sistema. Los autores concluyen que el sistema funciona adecuadamente en condiciones ideales donde no existe multitrayectoria, convirtiendo este fenómeno en el principal limitante del sistema y generando errores de hasta $30^{\circ}$ en la estimación con respecto a la posición real. 


\section{TDOA}

El segundo prototipo experimental presentado en (Stieber, 2012) está basado en TDoA, donde todo el procesamiento del algoritmo se realiza en un módulo T3-301, el cual tiene un procesador de 32 bits Coldfire. El firmware del dispositivo contiene todas las configuraciones de telemetría, reportes y se encarga de empaquetar los datos, y se conecta a un módulo GPS a través de una interfaz serial con el fin de contar con una fuente de sincronización externa. El sistema presenta buenos resultados, obteniendo resolución de 300 metros cuando se emplean al menos tres sensores del mismo tipo.

\section{E. Otras Técnicas}

En (Keaveny, 2005) los autores realizan el diseño y la implementación de un algoritmo de RDF de un solo canal sobre una plataforma SDR. Para realizar la estimación, el sistema anterior emplea algoritmos de AoA que usan los cambios de fase que se reflejan en el arreglo de antenas, con la restricción de sólo estar en capacidad de rastrear una señal que se encuentre entre -ð у , y obligando a realizar una calibración adicional del sistema que permita rastrear señales con otro rango de fase. Los resultados de las pruebas realizadas permiten concluir que el sistema tiene una precisión de $6^{\circ}$ respecto a la posición real, siempre y cuando se tengan $\mathrm{SNR}=10 \mathrm{~dB}$.

Como un complemento al trabajo presentado en (Keaveny, 2005), en (Harter, 2007) se realiza una calibración del sistema que corrige el desfase generado por el conmutador RF. Sin embargo, el funcionamiento del sistema es el mismo, ya que se hace un barrido del arreglo de antenas que permiten recibir la señal en cada uno de ellos en diferentes instantes de tiempo. Dichos datos se almacenan y posteriormente se emplean para el cálculo de la estimación, pasando de un sistema no lineal a uno lineal con el fin de reducir la complejidad del algoritmo y por lo tanto la carga computacional. Con el sistema descrito se realizan algunas pruebas adicionales que involucran movimiento de la fuente que se está tratando de localizar con el fin de evaluar su desempeño bajo estas circunstancias. Finalmente se concluye que siempre y cuando los movimientos sean lentos y pequeños, el prototipo desarrollado está en la capacidad de estimar la posición.

Las funciones de correlación cruzada son ampliamente usadas por los algoritmos de posicionamiento tal y como se muestra en (Balogh \& Kollar, 2003) dónde se emplea el principio de interferometría. En este tra- bajo se emplea un arreglo de cuatro elementos de antena dispuestas en forma de cuadrado que reciben la misma señal RF en diferentes posiciones, luego se comparan cada par de señales y se emplean funciones de correlación para obtener los máximos locales y de esta manera estimar el ángulo de llegada de señal transmitida.

\section{Implementaciones de técnicas de RDF en plataformas alternativas}

La arquitectura general de los sistemas desarrollados para RDF que utilizan FPGA o DSP, consiste en la integración de un arreglo de antenas, los circuitos de front-end, conversores A/D (análogo/digital) de múltiples canales y la plataforma de desarrollo en donde se programan o describen los algoritmos de estimación. Algunas aplicaciones utilizan circuitos de media frecuencia antes de los A/D y otros realizan la conversión a IF (Frecuencia intermedia) o banda base con digital down convertion (DDC) dentro del dispositivo de desarrollo.

\section{$3.1 F P G A$}

Los dispositivos lógico programables se han convertido en una fuerte alternativa para la implementación de sistemas de procesamiento de señales (DSP). Son plataformas heterogéneas que cuentan con diversos componentes de hardware, software e interconexión, que gracias a sus múltiples recursos, permiten la implementación de circuitos lógicos con grandes niveles de paralelismo y segmentación lo que permite generar una arquitectura muy eficiente para dar solución al problema en consideración.

Algunos autores exponen que el procesamiento basado en FPGA, se ha consolidado como una de las tecnologías más atractivas para desarrollar estimadores de DoA, dada la gran flexibilidad del hardware en adición con la habilidad de ejecutar algoritmos conjuntamente con el software. No obstante, la gran mayoría de autores realizaron modificaciones a los algoritmos, simplificando operaciones matemáticas o algebraicas para disminuir la complejidad de la implementación, la cantidad de recursos consumidos y los tiempos de ejecución.

En la gran mayoría de aplicaciones encontradas en la implementación de algoritmos de RDF utilizando FPGA, las pruebas del sistema se hacen con una fuente simulada y en ambientes donde se evita el fenómeno de multitrayectoria. 
Los desarrollados encontrados se muestran a continuación segmentados por el algoritmo de RDF que se emplea:

\section{A. Bartlett}

Comparaciones en la implementación de hardware y software del algoritmo Bartlett para la estimación de DoA fueron publicadas por Absolutan et al. (2010) y LaMeres et al. (2011), en donde se utilizó una tarjeta ML507 con una FPGA Virtex-5 FX70. El sistema consta de un arreglo circular de ocho antenas, cuyas señales se digitalizaban por medio de dos conversores A/D AD9287 de cuatro canales a $12.5 \mathrm{Msps}$, ensamblados en un circuito fabricado por los autores. Las ocho señales se procesan paralelamente en la FPGA. El algoritmo se implementa de dos maneras: en hardware con lógica combinacional y en software utilizando el procesador MicroBlaze ${ }^{\mathrm{TM}}$ embebido en la FPGA. Una comparación de los dos desarrollos muestra que el hardware es 300 veces más rápido que el software. Sin embargo, el tiempo de desarrollo del software es 4 veces menor que en hardware. Se observan iguales escalas de precisión en ambas soluciones, razón por lo cual los autores recomiendan una implementación híbrida del sistema.

\section{B. Técnicas Monopulso}

Ipek (2006) implementa dos técnicas monopulso para determinar el AoA en una Virtex-II Pro, con una frecuencia de reloj de $200 \mathrm{MHz}$ utilizando la herramienta de alto nivel de System Generator ${ }^{\mathrm{TM}}$ (SysGen), reportando que la FPGA es capaz de brindar una estimación en 120 ciclos de reloj.

En la primera etapa realiza una comparación en amplitud con un arreglo circular de 6 elementos de antena, para luego, en una segunda etapa, realizar la comparación en fase, empleando un arreglo de solo 2 elementos. Los resultados muestran que se obtienen errores de $5.2^{\circ}$ y $0.9^{\circ}$ respectivamente, con una $\mathrm{SNR}$ de $20 \mathrm{~dB}$.

\section{MUSIC}

Debido a que es un algoritmo de alta resolución, MUSIC es ampliamente utilizado. Este algoritmo requiere la estimación y la descomposición de los eigenvalores de la matriz de covarianza, haciéndolo computacionalmente pesado, lo cual dificulta su implementación sobre FPGA.

Algunos autores como Zou et al. (2006) proponen métodos para simplificar el algoritmo y poder realizar una descripción en hardware que cumpla con los requisitos de ejecución en tiempo real. Otros han empleado algoritmos con CORDIC (Fan, et al., 2011), (Zhou, et al., 2012), obteniendo desviaciones en la estimación menores a $0.5^{\circ}$.

En (Lounici \& Luan, 2013) y (Lounici, et al., 2013), el autor usó este algoritmo, y mediante SysGen expone un estudio de los recursos utilizados en la multiplicación de matrices necesaria para la obtención de los eigenvalores, lo cual ayuda a la elección de un dispositivo con la densidad adecuada.

En (Kim, et al., 2003), (Kim \& Arai, 2004) y (Kim \& Ichige, 2004), se exponen implementaciones enfocadas a la estimación DoA para antenas celulares. Los autores también emplean el algoritmo de Jacobi basado en CORDIC para simplificar la implementación del algoritmo.

Otras implementaciones de MUSIC fueron hechas por Wang et al. (2006) con un FPGA Virtex-II Pro XC2VP30, en donde se hace una estimación en $30 \mu \mathrm{s}$ utilizando un arreglo lineal de 4 antenas. Se realizan pruebas ubicando emisores a $-15^{\circ}$ y $20^{\circ}$ donde la plataforma desarrollado fue capaz de ubicarlos en $-14.97^{\circ}$ y $19.15^{\circ}$.

Por otra parte, mediante simulación se comparó el desempeño de tres arreglos de ocho antenas, ULA, rectangular(URA) y circular (UCA), para la técnica MUSIC (Miao \& Ying, 2009). Los resultados obtenidos muestran que para una señal con ángulo de $0^{\circ}$, el ULA arroja la peor estimación; mientras que para unángulo de $90^{\circ}$, ULA y URA muestran mejor desempeño. De los principales limitantes que se encontró tenía el ULA es la ambigüedad que se refleja mediante aparición de señales espejo, sin embargo, su desempeño es mejor que el del UCA.

En (Arai \& Ichige, 2005) los autores implementaron un procesador de MUSIC unitario (UMP). El sistema consta de un arreglo lineal de antenas, una serie de transceivers multicanal que se encargan de llevar la señal a banda base, conversores A/D a 20 Msps y 2 FPGA EP20K600. En una de ellas se encuentra el DDC y la extracción de la matriz de correlación, mientras que en la otra se hace la descomposición en eigenvalores y se hace la detección de mínimos locales. Con esta arquitectura se obtuvieron errores de $2^{\circ}$ para SNR de entrada mayores a $5 \mathrm{~dB}$.

\section{ESPRIT}

Una implementación de un algoritmo basado en ESPRIT es presentado en (Yaqoob, et al., 2011), donde 
los autores aprovechan las características del CSS IEEE802.15.4a para realizar las estimación del ángulo de llegada. El sistema emplea una FPGAAltera StartixII EP2S180 y dos transciever receptores MAX2830 EV. El algoritmo propuesto se verifica a través de simulación y de implementación práctica. La simulación se hace empleando un canal con ruido AWGN; mientras que las medidas experimentales se hacen con nodos trasmisores de CSS. Se obtienen resultados 5\% mejores con el algoritmo propuesto que cuando se aplica el algoritmo tradicional de ESPRIT.

\section{E. TDOA}

Una implementación de ToA y TDoA (Vandana, et al., 2012) es expuesta sobre una plataforma que consta de 4 sensores, los cuales envían sus medidas mediante una señal de video a una estación base que es compuesta por una Virtex-4. Se realizan comparaciones del desempeño del algoritmo simulando en Matlab.

\section{F. Interferometría De Fase}

Un sistema para estimar el AoA de aeronaves basado en interferometría es presentado por Guerin et al. (2012) utilizando una plataforma ML605 con una FPGA Virtex-6 LX240T, dos antenas direccionales tipo horn que proveen un rango de medida de $170^{\circ} \mathrm{y}$ conversores A/D en la tarjeta FMC110 a 250 Msps. El kit de desarrollo fue empleado para la adquisición de la señal y enviar los datos vía USB a un computador donde se ejecuta el algoritmo. Se realizan simulaciones en Matlab y en C para comparar el desempeño del sistema implementado, obteniendo errores no superiores a $0.415^{\circ}$ con una SNR de $40 \mathrm{~dB}$.

\section{G Otras Técnicas}

Un sistema que emplea cuatro elementos de antena es presentado en (Inserra \& Tonello, 2014). El sistema es empleado para comprar el desempeño del algoritmo de DoA cuando es simulado y en condiciones reales de implementación. Considerando los problemas que puede presentar el hardware y las restricciones del mismo, se realiza un conjunto de pruebas que permiten caracterizar y modelar el sistema con el fin de mejorar su desempeño, conociendo parámetros como ancho de banda, ganancia, ruido de fase, patrones de radiación de las antenas, entre otros. El algoritmo de DoA implementado busca correlacionar las señales que llegan a dos elementos de antena sucesivos, con el fin de emplear esta información en la estimación del ángulo de llegada de la señal que se trasmite.
El sistema está compuesto por un generador de señal Agilent que juega el rol del transmisor, una tarjeta rotativa para cuatro elementos de antena, cuatro circuitos de front-end para la conversión de señales de RF a banda base, una tarjeta de 8 canales equipada con 4 FPGA Xilinx Virtex y finalmente un PC controlador para el manejo de los dispositivos y encargado de todo el post-procesamiento de la información. Las antenas empleadas son dipolos cuya separación es de $6.2 \mathrm{~cm}$ entre cada par adyacente; y se emplea una frecuencia de portadora de $2.412 \mathrm{GHz}$.

Las pruebas para validar el desempeño del sistema se realizan dentro de una cámara anecoica y se obtienen buenos resultados para niveles de SNR superiores o iguales a $60 \mathrm{~dB}$, teniendo en cuenta que el hardware produce un error constante de $2.5^{\circ}$ en la estimación final.

Por otra parte, en (Garcia, et al., 2007) se presenta un sistema de RDF que emplea un arreglo lineal de sensores para calcular la dirección de llegada de una señal de interés. Los autores emplean un algoritmo de baja resolución para intentar ubicar dos fuentes interferentes, para lo cual emplean un modelo de canal que contempla efectos de multitrayectoria en el sistema y variaciones de SNR. Las pruebas arrojan resultados satisfactorios para trasmisores que estén separados al menos $14^{\circ}$ entre ellos, siendo posible identificar cada uno de ellos.

Finalmente, en (Peng, et al., 2012) se presenta un sistema de RDF que se prueba empleando un algoritmo SVR (Support Vector Regression) para mejorar su precisión. El sistema se prueba dentro de una cámara anecoica y está compuesto por una antena transmiso$\mathrm{ra}$, tres antenas para recepción, tres amplificadores, y tres módulos de filtros y divisores de potencia. Para la transmisión se emplea una antena tipo horn que está separada 1.4 metros del receptor. Los pruebas demuestran que al emplear el algoritmo SVR se obtienen resultados $0.2932^{\circ}$ mejores que cuando no se aplica este algoritmo.

\subsection{DSP}

Los DSP son dispositivos capaces de realizar operaciones matemáticas, trigonométricas y algebraicas a muy alta velocidad, además de que son fáciles de programar mediante lenguajes como $\mathrm{Co} \mathrm{C}++$, y cuentan con un gran número de librerías que facilitan el trabajo sobre ellos. Adicionalmente, muchos de ellos cuentan con múltiples núcleos y brindan la posibilidad de paralelizar el procesamiento, convirtiéndolos en una herramienta poderosa. 
Al igual que en las FPGA, en estos dispositivos se han implementado algoritmos de RDF, que estiman la posición de un emisor en tiempo real, mediante la simplificación o modificación de algunos métodos con el fin de disminuir los requerimientos de memoria del dispositivo.

DSP de Texas Instruments han sido empleados para la implementación de ESPRIT (Treiber, et al., s.f.), (Utschick, et al., 2000). Para las operaciones algebraicas se empleó un algoritmo basado en Jacobi que permitía encontrar los eigenvalores y eigenvectores. En total se alcanzan tiempos de estimación de $5 \mathrm{~ms}$ y $10 \mathrm{~ms}$, y escalas de precisión comparables a las herramientas de simulación.

Una aplicación enfocada a estimar el DoA en ambientes submarinos y su implementación con un DSP TMS320VC33 en un módulo VC33 de Texas Instruments es reportado por Hou et al. (2009). El sistema consta de un arreglo lineal de cuatro elementos y conversor A/D de 9 canales AD789. Esta plataforma se usó para comparar el desempeño MUSIC, Reduce-Order Root MUSIC, Spatial Smoothing MUSIC y Least-Squares, al ubicar una fuente acústica en $24.5^{\circ}$ a 152 metros. Los ángulos estimados fueron de $19.5^{\circ}$, $19.5^{\circ}, 20.2^{\circ}$ y $19.2^{\circ}$ respectivamente.

Se pueden encontrar otras implementaciones utilizando plataformas de Texas Instruments, como TMS320C6416T (Juncai, et al., 2008), en donde se desarrolla la estimación de DoA utilizando descomposición QR. Allí se propone un nuevo método basado en la transformada Householder la cual tiene un menor costo computaciones y emplea menos memoria. También se utiliza el DSP TMS320 (Wenke, et al., 2005) para encontrar el DoA en sistemas CDMA.

Una implementación del método Coherent Signal Subspace (CSS) fue desarrollado por Jamali et al. (2007) con un ULA de 16 elementos en un DSP DIOPSISTM 740 de Atmel de dos núcleos. El algoritmo fue implementado paralelamente y consta de 64 FFT de 64 puntos y el cálculo de 33 matrices de covarianza de $16 \times 16$. Esta implementación muestra igual desempeño comparada con implementaciones no paralelizadas con un menor tiempo de ejecución.

\subsection{Configuraciones Híbridas}

Este tipo de configuraciones son una alternativa que aprovecha la flexibilidad y velocidad del hardware y simplificando la implementación de algunas operaciones secuenciales con el procesador. En general, los cálculos de los algoritmos son divididos entre la FPGA que paraleliza el procesamiento de operaciones como la FFT; mientras que el DSP facilita la implementaciones de operaciones con matrices.

El empleo conjunto de FPGA y DSP es planteado por Li et al. (2013) con técnicas TDoA sobre un sistema de múltiples nodos sincronizados con GPS. Cada nodo consta de una antena, una FPGA y un DSP, conectados a una computadora central vía Ethernet que obtiene la información de todas las estaciones y realiza los cálculos para la estimación.

La FPGA se encarga de almacenar en una memoria DDR (Double Data Rate) los datos que llegan de los conversores $\mathrm{A} / \mathrm{D}$, los cuales tienen una frecuencia de muestreo de $60 \mathrm{MHz}$. Adicionalmente controlan el dispositivo GPS, mientras que el DSP se encarga de la conexión Ethernet con el computador central.

Por su parte Yao et al. (2008) desarrolló un sistema en FPGA y DSP en donde se implementa un algoritmo basado en la combinación de MUSIC y BeamForming (Multiple Sub-Array Beam-Space) denominado MSB-MUSIC, que reduce la complejidad y los requerimientos de SNR de MUSIC. La plataforma de hardware está conformada por 6 DSP TMS320C6713, 2 FPGA EP2C35 y un arreglo de 80 sensores. La primera FPGA se encarga de obtener las señales de los 80 canales y convertirlas en banda base utilizando CORDIC para ejecutar las operaciones trigonométricas en el DDC, y utiliza dos FFT de 128 puntos para obtener la representación de las señales en frecuencia. Luego 4 DSP son utilizados para calcular la matriz de covarianza y posteriormente se hace la descomposición en eigenvalores mediante CORDIC basado en el algoritmo de Jacobi paralelo en la segunda FPGA, para finalmente calcular el ángulo en los 2 DSP restantes. Esta plataforma se usa en ambientes subacuáticos, brindando alto rendimiento y buena precisión.

\subsection{Otras Plataformas}

Majid et al. (2013) presenta un trabajo en donde expone la paralelización del algoritmo MUSIC y su implementación en un procesador Intel Nehalem Quad Core, una NVidia GeForce GTX 260 GPU y un IBM Cell BE Processor, para un arreglo lineal de antenas de 16 elementos. Una de las pruebas realizadas fue evaluar el valor mínimo de muestras en la FFT para obtener resultados precisos. Se obtiene que para valores de 16, 32 y 64 puntos, la estimación es adecuada, pero para 8 puntos se presentan picos ex- 
tras que impidieron la estimación de la posición del emisor. La conclusión final arroja que la plataforma más eficiente en términos de tiempo de ejecución es la GPU de NVidia. Además resaltan que para este tipo de aplicaciones en donde se paraleliza el procesamiento, entre mayor sean los datos a procesar con un número menor de tareas, el tiempo computacional será menor al que se presenta con pocos datos y un número más elevado de tareas. Lo anterior es debido al exceso de procesos de comunicación que se deben realizar para coordinar las tareas.

Por otra parte, la plataforma PXI de National Instruments es utilizada para comprar por medio de LabView, tres algoritmos de DoA(Tayem, et al., 2013), MUSIC, ESPRIT y un nuevo método propuesto denominado QR-TLS. Se emplea un arreglo lineal de 4 antenas y se hacen pruebas en un techo donde se ubican dos emisores de manera simultánea en $64^{\circ}$ y $104^{\circ}$. Los algoritmos estiman los ángulos $64.38^{\circ}$ y $-104.65^{\circ}$, $64.46^{\circ} \mathrm{y}-104.48^{\circ}, 65.54^{\circ} \mathrm{y}-104.41^{\circ}$ respectivamente.

\section{Conclusiones}

Diversas implementaciones de algoritmos de RDF son expuestas, mostrando la posibilidad de su desarrollo en diferentes plataformas como son SDR, FPGA, DSP y procesadores de propósito general.

Algunas desventajas observadas en la implementación de sistemas embebidos como FPGA y DSP, son su mayor tiempo de desarrollo comparado con los SDR, debido a que los circuitos de front-end y controladores de ADS deben ser considerados para un diseño de este tipo, en cambio para plataformas SDR estos son transparentes en el momento de desarrollar la implementación, convirtiendo de manera automática la señal de RF a banda base.

Otro de los limitantes de las FPGA es la descripción de los algoritmos de medida, ya que exigen la simplificación y modificación de los métodos debido a la complejidad de la implementación de algunas operaciones matemáticas, matriciales y algebraicas que consumen recursos limitados de la misma. Además los lenguajes de descripción de hardware son de bajo nivel a diferencia de los empleados en plataformas SDR, donde se cuenta con librerías ya desarrolladas que facilitan el trabajo.

Uno de los principales factores por los cuales se ven en desventaja el uso de FPGA con respecto a los SDR en la aplicación de interés que son tareas de gestión del espectro en países en vía de desarrollo, es el costo del software de desarrollo, ya que las plataformas SDR cuentan con software libre como GNU y Python, y en las FPGA se deben adquirir herramientas de alto costo.

La recomendación final para la implementación de un sistema de RDF para tareas de gestión del espectro en países en vía de desarrollo donde se buscan sistemas más económicos, es emplear una plataforma SDR debido a su bajo costo y a la posibilidad de un rápido desarrollo del prototipo.

Las implementaciones en FPGA o DSP son una buena opción si se desea optimizar el tiempo de ejecución del algoritmo, ya que son herramientas que permiten ejecución de aplicaciones en tiempo real.

\section{Referencias bibliográficas}

Abusultan, M., Harkness, S., LaMeres, B. \& Huang, Y., 2010. $\{F P G A\}$ implementation of a $\{B\}$ artlett direction of arrival algorithm for a $5.8 \mathrm{ghz}$ circular antenna array. s.1., s.n., pp. 1-10.

Arai, H. \& Ichige, K., 2005. Hardware Implementation of Smart Antenna Systems for High Speed Wireless Communication. International Union of Radio Science, Proc. Of Gernal Assemblies.

Bahamondes, R., 2009. Digital Signal Processing. Tecnología Digitaly Microprocesadores \{@ONLINE\}. s.1.:s.n.

Balogh, L. \& Kollar, I., 2003. Angle of arrival estimation based on interferometer principle. s.1., s.n., pp. 219223.

EMT, F., 2004. RIMAX-A Flexible Algorithm for Channel Parameter Estimation from Channel Sounding Measurements.

Ettus, R., 2015. Ettus USRP N210. s.1.:s.n.

Fan, W., tao, G. H., Lin, Z. \& xiang, S. Y., 2011. Hardware Implementation of \{MUSIC\} Algorithm for Airborne Digital Direction Finding System. s.1., s.n., pp. 1-4.

Fette, B. A., 2006. Cognitive Radio Technology. s.1.:Communications Engineering Series.

Garcia, A., Mendez, A. \& Gonzalez, J., 2007. Fuzzy System for $\{D O A\}$ Estimation in Mobile 
Communications using a $\{F P G A\}$. s.1., s.n., pp. 14-14.

Gross, F., 2015. Smart antennas with matlab: principles and applications in wireless communication. s.l.: McGraw-Hill Professional.

Guerin, D., Jackson, S. \& Kelly, J., 2012. A Phase Interferometry Direction Finding System for an Airborne Platform, s.l.: s.n.

Harter, N. M., 2007. Development of a single-channel direction finding algorithm.

Hou, S.-Y., Chang, S.-H., Hung, H.-S. \& Chen, J.-Y., 2009. \{DSP $\}$-Based Implementation of a Real-Time \{DOA\} Estimator for Underwater Acoustic Sources. Journal of Marine Science and Technology, 17(4), pp. 320-325.

Hua, M.-C.y otros, 2011. Direction-of-arrival estimator using array switching on software defined radio platform. s.1., s.n., pp. 2821-2824.

Hua, M.-C., Hsu, C.-H. \& Liu, H.-C., 2012. Implementation of direction-of-arrival estimator on software defined radio platform. s.1., s.n., pp. 1-4.

Inserra, D. \& Tonello, A. M., 2014. A multiple antenna wireless testbed for the validation of $\{\mathrm{DoA}\}$ estimation algorithms. AEU - International Journal of Electronics and Communications, \#jan\#, 68(1), pp. 10-18.

ÍPek, A. V., 2006. Implementation of a Direction Finding Algorithm on an $\{F P G A\}$ Platform, s.l.: s.n.

ITU, 2011. Recomendación ITU-T K.83 Monitoring field strengths of electromagnetic fields, Ginebra: s.n.

ITU, s.f. Spectrum Management System for Developing Conuntries. En: s.1.:s.n.

Jamali, M. y otros, 2007. $\{D S P\}$ Based Implementation of Direction of Arrival for Wideband Sources. s.1., s.n., pp. $475-480$.

Juncai, S., Wenzhuo, G. \& Shu, Z., 2008. An improved method of $\{D O A\}$ estimation based on $\{Q R\}$ decomposition and its $\{D S P\}$ implementation. s.1., s.n., pp. 189-195.

Keaveny, J. J., 2005. Analysis and Implementation of a Novel Single Channel Direction Finding Algorithm on a Software Radio Platform.
Kim, M. \& Arai, H., 2004. Real-time smart antenna system incorporating $\{F P G A\}$-based fast $\{D O A\}$ estimator. s.l., s.n., pp. 160-164, Vol. 1.

Kim, M. \& Ichige, K., 2004. Implementation of \{FPGA Based Fast Unitary \{MUSIC DOA Estimator. IEICE TRANSACTIONS on Electronics, E87-C(9), pp. 1485-1494.

Kim, M., Ichige, K. \& Arai, H., 2003. Implementation of $\{F P G A\}$ based fast $\{D O A\}$ estimator using unitary \{MUSIC\} algorithm [cellular wireless base station applications]. s.l., s.n., (1), pp. 213-217.

LaMeres, B. y otros, 2011. Design and test of $\{F P G A\}-$ based direction-of-arrival algorithms for adaptive array antennas. s.l., s.n., pp. 1-8.

Li, Q. y otros, 2013. A hardware design for time delay estimation of $\{T D O A\}$. s.l., s.n., pp. 1-3.

Lounici, M., Luan, X. \& Saadi, W., 2013. Implementation of $\{\mathrm{QR}\}$-decomposition based on $\{\mathrm{CORDIC}\}$ for unitary $\{$ MUSIC $\}$. Fifth International Conference on Digital image Processing, Volumen 8878.

Lounici, M. W. \& Luan, X. M., 2013. Implementation of Unitary \{MUSIC $\}$ Algorithm Using $\{\mathrm{X}\}$ ilinx $\{\mathrm{S}\}$ ystem $\{\mathrm{G}\}$ enerator. Advanced Materials Research, Aug, Volumen 748, pp. 629-633.

Majid, M. W., Schmuland, T. E. \& Jamali*, M. M., 2013. Parallel implementation of the wideband $\{\mathrm{DOA}\}$ algorithm on single core, multicore, $\{\mathrm{GPU}\}$ and $\{\mathrm{IBM}\}$ cell $\{\mathrm{BE}\}$ processor. Science Journal of Circuits, Systems and Signal Processing, Volumen 2, pp. 29-36.

MEDAV, 2015. KCS 8000 MEDAV. s.1.:s.n.

Miao, S. W. \& Ying, Z. J., 2009. Research and \{FPGA\} Implementation of $\{$ MUSIC DOA $\}$ of Smart Antenna Array. s.l., s.n., pp. 189-192.

Navarro, A., s.f. Presentación del proyecto: Desarrollo de Capacidades Tecnológicas en Comprobación Técnica del Espectro. s.l.:s.n.

Peng, H., Yang, Z. \& Yang, T., 2012. Design and implementation of a practical direction finding receiver. Progress In Electromagnetics Research Letters, Volumen 32, pp. 157-167.

Roxin, A.-M., Gaber, J., Wack, M. \& Moh, A. N. S., 2007. Survey of wireless geolocation techniques. s.l., s.n., 9 pages. 
Series, S., 2011. Comparison of Time-Difference-ofArrival and Angle-of-Arrival Methods of Signal Geolocation.

Stieber, M. C. E., 2012. Radio Direction Finding Network Receiver Design for Low-Cost Public Service Applications, s.1.: s.n.

Tayem, N., Omer, M. \& Abul Hussain, A., 2014. Hardware Implementation of MUSIC and ESPRIT on NI-PXI Platform. s.1., s.n., pp. 329-332.

Tayem, N. y otros, 2013. Hardware Implementation of a Proposed $\{\mathrm{QR}-\mathrm{TLS}\}\{$ DoA $\}$ Estimation Method and $\{$ MUSIC $\}$, \{ESPRIT $\}$ Algorithms On \{NIPXI\} Platform. Progress In Electromagnetics Research C, Volumen 45, pp. 203-221.

Thanh, H. T., Ha, T. N. \& Van Yem, V., 2013. Novel direction finding algorithm based on phase locked loop with low computational complexity. s.l., s.n., pp. 437-442.

Treiber, M., juhn, T. K. \& Utschick, W., s.f. $\{\mathrm{DSP}\}-$ Implementation of a High-Resolution Parameter Estimating Scheme.

UIT, 2011. Manual de Comprobación Técnica del Espectro, Ginebra: Union Internacional de Telecomunicaciones, Oficina de Radiocomunicaciones: s.n.

Utschick, W., Treiber, M., Kurpjuhn, T. \& Nossek, J., 2000. Comparison of two $\{D O A\}$ tracking implementations for $\{S D M A\}$. s.l., s.n., (1), pp. 358-362.

Van Trees, H. L., 2004. Detection, estimation, and modulation theory, optimum array processing. s.1.:John Wiley $\backslash \&$ Sons.

Vandana, M., Rani, S. \& Badrinath, 2012. Radar Emitter Location Simulation and Implementation on \{FPGA . International Journal of Engineering and Innovative Technology (IJEIT), Oct. Volumen 2.
Wang, H. \& Glesner, M., 2006. Hardware implementation of smart antenna systems. Advances in Radio Science, Volumen 4, pp. 185-188.

Wenke, L., liang, J. \& Chenxin, M., 2005. \{DOA\} estimation of $\{C D M A\}$ signals and the realization utilizing \{TMS320\}. s.1., s.n., pp. 561-564.

XILINX, s.f. Field Programmable Gate Array $\{@ O N L I N E\}$. s.1.:s.n.

Yao, B. y otros, 2008. Real-Time Implementation of Multiple Sub-array Beam-Space \{MUSIC\} Based on $\{F P G A\}$ and $\{D S P\}$ Array. s.l., s.n., pp. 186191.

Yaqoob, A., Farooq, U., Abbas, G. \& Asad, M. U., 2011., Efficient Hardware Implementation Of ESPIRIT like Algorithm for Range Estimation Of Chirp Spread Spectrum. International Journal Of Computer and Electrical Engineering, 3(0).

Zekavat, R. \& Buehrer, R. M., 2011. Handbook of position location: Theory, practice and advances. s.1.:John Wiley $\backslash \&$ Sons.

Zekavat, S. y otros, 2007. A novel implementation of doa estimation for node localization on software defined radios: Achieving high performance with low complexity. s.l., s.n., pp. 983-986.

Zhou, L. y otros, 2012. Design and implementation of equilateral triangle array digital direction finding system. Electronics Express, 9(13), pp. 1102-1108.

Zou, Z., Hongyuan, W. \& Guowen, Y., 2006. An Improved \{MUSIC\} Algorithm Implemented with High-speed Parallel Optimization for $\{F P G A\}$. s.l., s.n., pp. 1-4. 
